\title{
Validade discriminante entre regulação emocional e resiliência
}

\author{
Angélica Maria Ferreira de Melo Castro ${ }^{1}$, José Maurício Haas Bueno, Mirela Dantas Ricarte, Emily Souza Gaião e Albuquerque \\ Universidade Federal de Pernambuco, Recife-PE, Brasil
}

\section{RESUMO}

Resiliência e regulação de emoções (RE) são construtos psicológicos cujas operacionalizações nunca foram investigadas quanto à independência mútua. Por isso, objetivou-se buscar evidências de validade discriminante entre medidas de resiliência e de RE. Para isso, foram aplicadas versões online do Inventário de Competências Emocionais (ICE-R) e da Escala de Resiliência para Adultos (RSA) em 169 participantes com média de idade de 29,8 anos, sendo 65,7\% do sexo feminino. Resultados de análise fatorial exploratória revelaram que os fatores primários de resiliência e RE tendem a se agrupar em fatores distintos de segunda ordem. Ainda que a "percepção de si" tenha apresentado carga nos dois fatores de segunda ordem, tais resultados sugerem que as medidas são predominantemente independentes entre si.

Palavras-chave: regulação emocional; resiliência; validade discriminante.

\section{ABSTRACT - Discriminant validity between emotional regulation and resilience}

Resilience and emotional regulation (ER) are psychological constructs whose operations have never been investigated in terms of mutual independence. Therefore, this study searched for evidence of discriminant validity between measures of resilience and ER. Thus, online versions of the Emotional Competence Inventory (ICE-R) and the Resilience Scale for Adults (RSA) were applied to 169 participants with mean age of 29.8 years, predominantly female $(65.7 \%)$. The results of an exploratory factorial analysis revealed that primary factors of resilience and regulation of emotions tend to cluster in different second-order factors. Even if "self-perception" had cross-loaded in both second-order factors, these results suggest that the measures are predominantly independent of one another. Keywords: emotional regulation; resilience; discriminant validity.

\section{RESUMEN - Validez discriminante entre regulación emocional y la resiliencia}

La resiliencia y la regulación emocional son constructos psicológicos cuya operacionalización nunca fue investigada por la independencia mutua. Por lo tanto, el objetivo de este estudio fue buscar evidencias de validad discriminante entre las medidas de resiliencia y regulación emocional. Para esto, se aplicaron versiones online del Inventario de Competencias Emocionales (ICE-R) y de la Escala de Resiliencia para Adultos (RSA) en 169 participantes con una edad media de 29,8, de los cuales un 65,7\% eran mujeres. Los resultados del análisis factorial exploratorio reveló que los factores primarios de resiliencia y regulación emocional tienden a agruparse en diferentes factores de segundo orden. Aunque la "percepción de sí mismo" ha presentado carga en los dos factores de segundo orden, estos resultados sugieren que las medidas son en gran parte independientes entre sí.

Palabras clave: regulación emocional; resiliencia; validez discriminante.

As emoções têm sido alvo frequente de interesse e estudos em diversas áreas do conhecimento científico. Elas surgem como processos centrais no funcionamento humano, desempenhando papel importante como organizadoras do desenvolvimento cerebral e em diversos domínios do funcionamento psicológico e social (Kemper, 2004). As pessoas são constantemente confrontadas com situações em que alguma emoção se encontra presente, por isso torna-se relevante aprender a lidar com elas e adquirir hábitos emocionais saudáveis.
Desde muito tempo, a regulação emocional (RE) ocupa lugar importante na investigação sobre emoções, tendo sido abordada nos estudos de Darwin (1872), segundo os quais certas formas de expressão emocional se baseiam em seus aspectos funcionais e regulatórios. $\mathrm{Na}$ Psicologia, o tema foi investigado sob diferentes perspectivas, como nos estudos sobre a teoria tridimensional das emoções de Wundt (1863), no campo da Psicanálise (Freud, 1946) e nas pesquisas sobre estresse e coping (Lazarus, 1996). Mais recentemente, a RE foi proposta como uma das habilidades relacionadas à inteligência 
emocional (IE) (Salovey \& Mayer, 1990; Mayer, Caruso, \& Salovey, 2016), embora ainda sem aporte teórico robusto (MacCann \& Roberts, 2008), o que veio a ocorrer com os estudos de Gross $(2008,2015)$.

Nas últimas décadas, a investigação sobre RE tem adquirido maturidade e se constituído em uma área de investigação científica, além do notável reconhecimento de sua importância para o desenvolvimento dos aspectos saudáveis do ser humano (Harris, Hare, \& Rangel, 2013). Ela é definida como a administração das emoções para promover o crescimento emocional e intelectual (Mayer et al., 2002), tornando-se essencial para manter o equilíbrio do sujeito (Gross, 2015), permitir relações adaptadas e promover a saúde mental (Cruvinel \& Boruchovitch, 2011).

Para mensurar as habilidades que compõem a IE percepção das emoções, utilização da emoção como facilitadora do pensamento, compreensão e análise de emoções e RE -, Mayer e Salovey (1997) construíram a Multifactor Emotional Intelligence Scale (MEIS). Uma revisão da MEIS deu origem ao Mayer-Salovey-Caruso Emotional Intelligence Test (MSCEIT) (Mayer et al., 2002), o instrumento mais empregado em estudos envolvendo a inteligência emocional.

Nesse contexto, a RE se refere à capacidade de tolerar reações emocionais agradáveis ou desagradáveis, compreendendo-as sem exageros ou diminuição de sua importância, e aprendendo a controlá-las ou descarregá-las apropriadamente. Porém, é somente a partir do reconhecimento do humor e da abertura aos sentimentos que as emoções finalmente podem ser compreendidas e, assim, separadas conscientemente do comportamento (Mayer \& Salovey, 1997).

Dessa forma, a RE pode ser conceituada como um construto que envolve a percepção, a expressão e a compreensão das emoções; a habilidade para facilitar os pensamentos e comportar-se em concordância com os objetivos pretendidos e a capacidade em utilizar, de forma flexível, estratégias de RE situacionalmente apropriadas para modular as respostas emocionais (Gross, 2015).

Assim, pode-se afirmar que a RE consiste no processo por meio do qual o indivíduo procura influenciar as situações em que as emoções são desencadeadas, os processos psicológicos que as desencadeiam (como a atenção e a percepção, por exemplo) ou a forma como serão (ou não) expressas pelo comportamento (Gross, 2008, 2015). A utilização de tais estratégias visa aumentar, diminuir ou manter os componentes fisiológicos, cognitivos e/ou comportamentais de uma resposta emocional, de modo a conciliar demandas pessoais e sociais (Gross, 2002). Com isso, a competência na utilização dessas estratégias está diretamente relacionada ao sucesso da adaptação ao contexto, tendo como consequência, por exemplo, a melhoria da qualidade de vida (Bueno, Correia, Abacar, Gomes, Pereira Junior, 2015) e a redução ou evitação do estresse (Schneider, Lyons, \& Khazon, 2013).
Outro construto da ciência psicológica que explora as diferenças individuais no enfrentamento de adversidades ao longo do desenvolvimento humano é a resiliência (Masten, 2013). O interesse dos pesquisadores nessa temática iniciou-se a partir da percepção de que acontecimentos hostis não tinham como consequência, obrigatoriamente, problemas psíquicos e comportamentais relacionados nos indivíduos que os experienciavam, uma vez que alguns deles demonstravam estar bem, apesar do contexto de risco (Cicchetti, 2013; Evans, Li, \& Whipple, 2013; Mayer, Salovey, \& Caruso, 2002).

Pesquisas nessa temática buscam compreender diferentes formas de adaptação que as pessoas apresentam aos acontecimentos no curso da vida (Overton, 2013). Atualmente, sabe-se que a resiliência apresenta um papel importante nesse processo adaptativo e de superação (Reppold, Mayer, Almeida, \& Hutz, 2012). Esse construto foi definido como a capacidade de suporte e adaptação bem-sucedida de um sistema dinâmico diante de uma perturbação significativa que ameace seu desenvolvimento, sua viabilidade ou sua função. Tal sistema pode ser considerado a pessoa, a família, o sistema de segurança, a economia, entre outros. Por esse motivo, a resiliência tem sido amplamente pesquisada nas mais diversas áreas do conhecimento (Masten, 2014).

Esse construto é originário das ciências exatas, mais especificamente dos campos da física e da engenharia, referindo-se à capacidade de uma matéria em retornar à forma ou posição inicial depois de submetida a uma ação extrema de energia, mesmo que esta tenha provocado algum tipo de deformação (Knorst, 2012). Na Psicologia, sua definição tem passado por modificações (Carvalho, Teodoro, \& Borges, 2014), porque, diferentemente dos materiais, uma pessoa não volta ao seu estado inicial depois de vivenciar um evento estressor. Essa experiência se agrega às anteriores, gerando mudanças e permitindo o desenvolvimento e amadurecimento psíquico, social e cognitivo (Paula Couto, Poletto, Paludo, \& Koller, 2006). Em outras palavras, no campo psicológico a resiliência diz respeito à capacidade de uma pessoa superar vivências negativas com relativo sucesso (Reppold et al., 2012), o que não implica necessariamente na eliminação do problema, mas em sua ressignificação (Junqueira \& Deslandes, 2003).

O significado atribuído pela pessoa a determinada situação é elaborado em conjunto com outras experiências pessoais que ela possui (Paludo \& Koller, 2005). Tais vivências possuem relação estreita com três categorias abrangentes, características da resiliência:

1. atributos disposicionais positivos individuais, que incluem sistemas cerebrais de aprendizagem, sistemas de controle motivacional, de resposta ao estresse e de autorregulação;

2. suporte familiar envolvendo a parentalidade, dinâmicas interpessoais, coesão, rituais e normas; e 
3. sistemas de suporte externos à família, como sistemas de pares, escolar, cultural e social (Masten \& Obradovic, 2006).

Dito isso, percebe-se que a resiliência pode ser considerada uma resposta positiva a fatores de risco em que há um processo adaptativo. Esses fatores estão vinculados a todo tipo de evento negativo na vida de uma pessoa e aumentam as chances do aparecimento de problemas físicos, psicológicos, comportamentais e sociais (Koller, Morais, \& Cerqueira-Santos, 2009).

Dessa forma, pelo fato de a RE estar relacionada ao crescimento emocional e intelectual, referindo-se à capacidade de tolerar reações emocionais agradáveis ou desagradáveis, é plausível supor que o processo de resiliência esteja relacionado com a RE. Tugade e Fredrickson (2007) parecem apoiar essa tese quando afirmam que pessoas resilientes tendem a fazer uso de emoções positivas enquanto pensam de forma estratégica, cultivando-as para lidar com fatores estressantes. De fato, há indicativos de que tanto a RE quanto a resiliência estão associadas a diferentes tipos de apego e influenciam o bem-estar psicológico (Karreman \& Vingerhoets, 2012). Além disso, pesquisadores observaram que certas estratégias de RE, como a refocalização no planejamento, a reavaliação positiva de eventos e a diminuição da ruminação, estão relacionadas com alta resiliência em pacientes com depressão e ansiedade (Min, Yu, Lee, \& Chae, 2013). Em um estudo de imageamento cerebral com mulheres vítimas e não vítimas de violência sexual (com e sem sintomas de estresse pós-traumático), foi observado que as vítimas que não apresentavam sintomas de estresse pós-traumático reagiam cerebralmente de forma semelhante às não vítimas de violência sexual. Já aquelas que apresentavam sintomas de estresse pós-traumático tinham muita dificuldade de regular emoções negativas. Para explicar o fenômeno observado, os autores levantaram a hipótese de que a habilidade de regular respostas frente a experiências negativas seja o mecanismo da resiliência para lidar com a exposição a traumas (New et al., 2009).

Gross (2015), por exemplo, diz que uma emoção pode ser regulada antes mesmo do contato com o estímulo que a dispara, pela seleção de uma situação emocionalmente mais favorável (seleção da situação), pela modificação da situação disparadora de modo a atenuar o efeito ou a intensidade do estímulo e, consequentemente, da reação emocional (modificação da situação), pela direção da atenção a aspectos ambientais mais neutros (redirecionamento da atenção), pela busca em reinterpretar a situação de modo a gerar uma emoção mais favorável (mudança cognitiva) ou pelo controle da resposta comportamental, apesar de se estar tomado internamente pela emoção (modulação da resposta). Usando apenas uma das cinco estratégias ou uma combinação dessas, seria possível controlar a intensidade das emoções e, consequentemente, melhorar a adequação do comportamento às exigências sociais envolvidas em determinada situação.

Assim, os dados apresentados parecem confirmar a impressão de que pessoas com alta capacidade de regular emoções (especialmente por meio da modificação cognitiva) devem ser mais resilientes após exposição ao estresse do que pessoas com baixa capacidade de regular emoções (Troy \& Mauss, 2011). Mais do que isso, a RE poderia ser considerada um importante meio pelo qual uma pessoa consegue se recuperar de uma experiência traumática, estressante ou, de alguma forma, perturbadora, como sugere o estudo de New et al. (2009). Dito de outra forma, a habilidade de regular emoções pode ser algo tão crucial ao processo resiliente a ponto de ser a própria resiliência. Embora alguns estudos sugiram que há relação entre esses dois construtos, até onde se pode levantar na literatura científica não há estudos que tenham se interessado por investigar a independência entre eles.

Nesse sentido, a problemática da presente pesquisa consistiu em analisar se a RE e a resiliência podem ser consideradas construtos distintos e independentes entre si. Do ponto de vista psicométrico, este trabalho se constituiu em um estudo de validade discriminante entre medidas de RE e de resiliência.

\section{Método}

\section{Participantes}

Trata-se de uma pesquisa com delineamento transversal, na qual os participantes foram abordados por meio de redes sociais ou contatados por e-mail. Assim, este estudo foi constituído por uma amostra não probabilística, por conveniência. Participaram desta pesquisa 169 sujeitos, sendo $33,7 \%$ do sexo masculino e $65,7 \%$ do sexo feminino ( $0,6 \%$ não informaram o sexo), com idades variando entre 16 e 62 anos (média $=29,8 ; D P=8,98$ anos). Nessa amostra, $86,9 \%$ eram residentes na região Nordeste, $8,9 \%$ na região Sudeste, $3,0 \%$ na região Centro-Oeste e 1,2\% na região Sul. Quanto à escolaridade, 55,1\% possuíam curso superior, 39,6\% eram estudantes do Ensino Superior e 5,3\% tinham concluído o Ensino Médio.

\section{Instrumentos}

A coleta de dados foi realizada por meio de três instrumentos: um questionário sociodemográfico, para caracterização da amostra; a versão revisada do Inventário de Competências Emocionais (ICE-R) (Correia \& Bueno, 2014) e a versão adaptada para o português brasileiro da Escala de Resiliência para Adultos (RSA) (Hjemdal, Roazzi, Dias, Roazzi, \& Vikan, 2009). O questionário socioeconômico recolheu informações pessoais (sexo, idade e escolaridade), econômicas (nível socioeconômico) e geográficas/culturais (local de moradia). 


\section{Inventário de Competências Emocionais}

A versão revisada do ICE-R é composta por 34 afirmações, para serem avaliadas por meio de uma escala Likert de 5 pontos, em que 1 significa "absolutamente não se aplica ao meu caso" e 5 significa "se aplica perfeitamente ao meu caso". O respondente também poderia escolher valores intermediários $(2,3$ e 4$)$ entre esses dois extremos, caso considerasse mais pertinente. Uma análise fatorial exploratória (AFE) indicou estrutura fatorial de cinco fatores:

1. RE em outras pessoas: envolve a habilidade para lidar com pessoas emocionalmente problemáticas ou situacionalmente tomadas pela emoção, sem desanimá-las ou constrangê-las, mas ajudando-as a se sentirem melhor e a darem o melhor de si;

2. RE de baixa potência em si mesmo: habilidade de não se deixar abater pela tristeza, desânimo, melancolia ou medo, sendo capaz de superar as frustrações do dia a dia;

3. expressividade emocional: habilidade para expressar-se emocionalmente, tanto do ponto de vista da comunicação dos sentimentos, quanto do desbloqueio da vergonha ou do medo de não aceitação por outras pessoas;

4. percepção de emoções: envolve a capacidade de perceber estados e alterações emocionais em si e em outras pessoas; $\mathrm{e}$

5. RE de alta potência em si mesmo: diz respeito à regulação da impulsividade, tanto da raiva quanto da euforia.

As consistências internas foram avaliadas pelo coeficiente alfa de Cronbach e variaram de 0,694 (percepção de emoções) a 0,866 (RE em outras pessoas) (Correia \& Bueno, 2014). Neste estudo, foram empregados apenas os três fatores que medem nuances da RE.

\section{Escala de Resiliência para Adultos}

A RSA foi desenvolvida por Hjemdal, Friborg, Martinussen, e Rosenvinge (2001) e adaptada para o português por Hjemdal et al. (2009). O instrumento apresenta 33 itens que são respondidos em uma escala de 7 pontos em formato de diferencial semântico. Uma análise de escalonamento multidimensional (MDS) revelou uma estrutura com seis fatores:

1. percepção de self: aponta o quanto as pessoas confiam nas capacidades que possuem; a visão positiva e realista que têm de si mesmas; e a percepção de autoeficácia;

2. planejamento do futuro: avalia o quão otimista é a visão de futuro dos participantes; o nível de segurança que sentem quanto à sua habilidade em criar e estabelecer metas bem delineadas e alcançáveis;

3. competência social: relacionada com a habilidade de iniciar diálogos e estabelecer novas amizades; com a maleabilidade nas relações sociais; com o sentimento de bem-estar em circunstâncias de socialização; e com a presença de um estilo de interação social;
4. estilo estruturado: diz respeito à capacidade de gerenciamento do tempo; do estabelecimento de metas e prazos; e da condução pessoal para manutenção de regras e rotinas no dia a dia;

5. coesão familiar: indica a qualidade das relações com membros da família em relação à comunhão de valores e visão de futuro, vínculo, lealdade e simpatia; 6. recursos sociais: englobam o apoio recebido por pessoas que estão fora do contexto familiar, as quais oferecem incentivo e ajuda em situações de dificuldade e compartilham sentimento de pertencimento e simpatia (Hjemdal et al., 2009).

Os índices de consistência interna, medidos pelo coeficiente alfa de Cronbach, variaram de 0,56 ("estilo estruturado") a 0,79 (“coesão familiar"). Embora o fator "estilo estruturado" apresente índice de precisão abaixo do valor mínimo recomendado na literatura cientifica $(0,70)$, optou-se pela utilização dessa escala em razão da insuficiência de instrumentos psicológicos para avaliação da resiliência que tivessem sido validados no Brasil. Ressalva-se, no entanto, que os resultados decorrentes dessa subescala da RSA devem ser analisados com cautela, pois as diferenças individuais nesse fator podem advir mais de erros aleatórios de medida do que de diferenças reais no construto avaliado.

\section{Procedimentos de pesquisa}

O projeto desta pesquisa foi submetido e aprovado por um Comitê de Ética em Pesquisa com Seres Humanos da Universidade Federal de Pernambuco, sob o protocolo CAAE n. ${ }^{\circ}$ 39406214.7.0000.5208. Dessa forma, seguiram-se todas as recomendações do Conselho Nacional de Saúde constantes na Resolução n. ${ }^{\circ}$ 466/2012 para a realização de pesquisas envolvendo seres humanos. Aqueles que concordaram em participar expressaram sua vontade habilitando uma opção no Termo de Consentimento Livre e Esclarecido (TCLE) do questionário virtual e, em seguida, respondendo aos instrumentos utilizados neste estudo, na seguinte ordem: questionário socioeconômico, RSA e ICE-R. Os dados coletados foram automaticamente armazenados em um banco de dados por meio da plataforma Google Docs.

\section{Análise dos dados}

A análise dos dados foi realizada com o auxílio do programa Statistical Package for the Social Sciences (SPSS). Para investigar a validade discriminante entre as medidas de resiliência e RE, empregou-se uma análise fatorial exploratória, com extração dos fatores por eixos principais e rotação oblíqua oblimin, sobre as pontuações dos participantes nos seis fatores primários do RSA e nos três de RE do ICE-R. A validade discriminante seria apoiada pela obtenção de dois fatores de segunda ordem, sendo um com os fatores primários de resiliência e outro com os de RE. Também são apresentados os índices de correlação entre os fatores primários de ambos os instrumentos. 


\section{Resultados}

Os dados são apresentados na sequência em que foram analisados. Primeiramente, são apresentadas as estatísticas descritivas dos dois instrumentos. Depois, são apresentados os resultados da análise fatorial de segunda ordem e, por fim, os coeficientes de correlação entre os fatores primários dos dois instrumentos. As análises descritivas da amostra nos instrumentos podem ser observadas na Tabela 1.

Pode-se observar que as médias de todos os fatores ficaram pouco acima do ponto central de suas escalas de resposta, o que provavelmente reflete o fato de ambos os construtos serem valorizados socialmente. Apesar disso, não se observou tendência acentuada de topo ou de base nas escalas. Também houve boa variabilidade dos escores em relação à média, indicando que os instrumentos captaram diferenças individuais nos construtos avaliados.

Para a verificação da validade discriminante entre as medidas de resiliência e de RE foi realizada uma AFE, com extração dos fatores por fatoração dos eixos

Tabela 1

Média e desuio-padrão nos fatores de regulação de emoções e resiliência

\begin{tabular}{lccc}
\hline Construtos & Fatores & Média & DP \\
\hline \multirow{4}{*}{ RE } & RE em outras pessoas & 3,70 & 0,69 \\
& RE de baixa & 3,48 & 0,78 \\
& potência em si & & \\
$\quad$ RE de alta & 3,44 & 0,66 \\
& potência em si & & \\
& Percepção de si mesmo & 4,90 & 1,20 \\
& Futuro planejado & 5,42 & 1,26 \\
Resiliência & Competência social & 5,24 & 1,23 \\
& Estilo estruturado & 4,82 & 1,24 \\
& Coesão familiar & 5,07 & 1,37 \\
& Recursos sociais & 5,72 & 1,03 \\
\hline
\end{tabular}

Nota: DP=desvio-padrão; RE=regulação emocional. principais e rotação oblíqua oblimin. As cargas fatoriais são apresentadas na Tabela 2.

As cargas fatoriais superiores a 0,4 foram consideradas mais significativas para interpretação (Hair, Black, Babin, Anderson, \& Tatham, 2009) e aparecem acompanhadas de asterisco (*) na Tabela 2. Dessa forma, observa-se que, de modo geral, os fatores primários de resiliência se agruparam no primeiro fator de segunda ordem (FSO1) e os de RE, no segundo (FSO2). No entanto, o fator primário "percepção de si mesmo", proveniente da escala de resiliência, apresentou carga fatorial similar em ambos os fatores de segunda ordem, sendo ligeiramente mais elevada no segundo fator.

Adicionalmente, com vistas a compreender melhor a relação entre resiliência e RE, realizou-se uma análise de correlação entre os fatores primários dos respectivos instrumentos de medida. Os resultados são apresentados na Tabela 3.

De forma geral, observa-se uma matriz de correlações positivas, quase todas estatisticamente significativas. No entanto, as correlações dos fatores primários relacionados ao mesmo construto (resiliência ou RE) são moderadas entre si (entre 0,4 e 0,7 ), enquanto as correlações

Tabela 2

Matriz de cargas fatoriais

\begin{tabular}{lccc}
\hline Construtos & Fatores primários & FSO1 & FSO2 \\
\hline \multirow{4}{*}{ Resiliência } & Recursos sociais &, $946^{*}$ &,- 129 \\
& Coesão familiar &, $688^{*}$ &,- 093 \\
& Estilo estruturado &, $453^{*}$ &, 137 \\
& Futuro planejado &, $446^{*}$ &, 290 \\
& Percepção de si mesmo &, $466^{*}$ &, $490^{*}$ \\
& RE de alta potência &,- 141 &, $817^{*}$ \\
RE & RE em outras pessoas &, 076 &, $696^{*}$ \\
& RE de baixa potência &, 128 &, $684^{*}$ \\
\hline
\end{tabular}

Nota: $\mathrm{FSO}=$ fator de segunda ordem; ${ }^{*}$ cargas fatoriais $>0,4$; $\mathrm{RE}=$ regulação emocional.

Tabela 3

Coeficientes de correlação $r$ de Pearson entre os fatores da regulação emocional e da resiliência

\begin{tabular}{|c|c|c|c|c|c|c|c|c|}
\hline & 1 & 2 & 3 & 4 & 5 & 6 & 7 & 8 \\
\hline 1 - RE em outras pessoas & 1 & & & & & & & \\
\hline 2 - RE de baixa potência & $0,561^{* *}$ & 1 & & & & & & \\
\hline 3 - RE de alta potência & $0,607^{* *}$ & $0,548^{* *}$ & 1 & & & & & \\
\hline 4 - Percepção de si mesmo & $0,480^{* *}$ & $0,617^{* *}$ & $0,428^{* *}$ & 1 & & & & \\
\hline 5 - Futuro planejado & $0,341^{* *}$ & $0,351^{* *}$ & $0,288^{* *}$ & $0,653^{* *}$ & 1 & & & \\
\hline 6 - Competência social & $0,362^{* *}$ & $0,372^{* *}$ & $0,174^{*}$ & $0,584^{* *}$ & $0,440^{* *}$ & 1 & & \\
\hline 7 - Estilo estruturado & $0,237^{* *}$ & $0,246^{* *}$ & $0,234^{* *}$ & $0,415^{* *}$ & $0,503^{* *}$ & $0,350^{* *}$ & 1 & \\
\hline 8 - Coesão familiar & $0,235^{* *}$ & $0,284^{* *}$ & 0,126 & $0,381^{* *}$ & $0,231^{* *}$ & $0,429^{* *}$ & $0,278^{* *}$ & 1 \\
\hline 9 - Recursos sociais & $0,334^{* *}$ & $0,328^{* *}$ & $0,177^{*}$ & $0,478^{* *}$ & $0,431^{* *}$ & $0,625^{* *}$ & $0,432^{* *}$ & $0,691^{* *}$ \\
\hline
\end{tabular}

Nota: RE=regulação emocional; ${ }^{*} p<0,05$ (bi-caudal); ${ }^{* *} p<0,01$ (bi-caudal). 
entre os fatores primários de ambos os construtos foram mais baixas (de 0,1 a 0,4). A exceção, novamente, foi a "percepção de si mesmo", que se correlacionou moderadamente tanto com os outros fatores de resiliência quanto com os de RE.

\section{Discussão}

O objetivo deste trabalho foi buscar evidências de validade discriminante entre medidas de resiliência e de RE. Para isso, foi empregada a análise fatorial, que consiste em um recurso estatístico capaz de detectar regularidades nos desempenhos (escores) das pessoas em diferentes tipos de tarefa. A tarefa proposta aos participantes desta pesquisa foi avaliar afirmações quanto ao modo de regular emoções (RE) e quanto aos seus recursos para se recuperar de eventos estressores (resiliência). Seus escores foram submetidos à análise fatorial, que os agrupa com base na concomitância com que variam, isto é, escores cujos valores variam concomitantemente (de um participante para outro) tendem a ser colocados em um mesmo fator. A ocorrência de um agrupamento é explicada por uma variável — ou traço latente — que não pode ser diretamente mensurada, mas subjaz a todos os componentes do fator (Primi, 2003).

Por isso, a análise fatorial pode ser empregada para verificação da validade discriminante entre resiliência e RE. A formação de apenas um fator de segunda ordem com cargas de todos os fatores primários sugeriria a existência de apenas um traço subjacente, indicando não haver discriminação entre resiliência e RE. Já a formação de dois fatores de segunda ordem indicaria a existência de dois traços latentes distintos.

Assim, os resultados da análise fatorial permitem constatar que, de fato, houve a separação dos fatores primários em dois fatores de segunda ordem, respectivamente relacionados à resiliência e à RE. Tal achado é compatível com a hipótese de construtos psicológicos distintos, tal como sugere a literatura científica (Gross, 2008; Masten \& Obradovic, 2006; Mayer et al., 2016; Reppold et al., 2012).

Ao lado disso, a independência entre as medidas em análise é corroborada pelos resultados das análises de correlação entre os fatores que constituem a resiliência e a RE. O fato das correlações terem sido moderadas entre fatores primários de cada construto (de 0,4 a 0,7 ) e baixas entre os construtos (de 0 a 0,4$)$ mostra que há mais variância comum entre os fatores primários relacionados ao mesmo construto do que entre fatores primários de construtos diferentes. Esse resultado, portanto, apoia a interpretação de que os construtos são, ao menos predominantemente, independentes, embora se correlacionem positivamente entre si.

Entretanto, apesar de serem esperadas cargas apenas entre os fatores de resiliência, observou-se que o fator primário "percepção de si mesmo" apresentou cargas similares nos dois fatores de segunda ordem, com valor ligeiramente maior no fator geral de resiliência $(0,490)$ em relação ao de RE $(0,466)$. Pode-se levantar duas hipóteses explicativas para essa ocorrência. Uma delas se dá por meio da IE, que engloba tanto a RE quanto a percepção de emoções. Esta última é definida como a capacidade de identificar emoções em si mesmo, em outras pessoas e em ambientes físicos e se relaciona com a RE e com outras habilidades que compõem a IE (MacCann, Joseph, Newman, \& Roberts, 2014; Mayer et al., 2016). Já a percepção de si mesmo, tal como avaliada pela RSA, é uma medida de como a pessoa percebe suas capacidades para lidar com imprevistos, solucionar problemas pessoais, julgar e tomar decisões, podendo ser entendida como uma medida de autoeficácia para lidar com as dificuldades da vida (Carvalho et al., 2014). Embora as definições de percepção de emoções e de percepção de si mesmo sejam distintas, elas envolvem o mesmo processo cognitivo básico: a percepção, que pode ser o processo comum por trás das relações de ambos os tipos de percepção com a RE.

A outra hipótese envolve a relação direta entre RE e percepção de si mesmo. Embora as análises correlacionais não permitam conclusões confiáveis sobre relações causais, é possível especular sobre a influência mútua entre esses dois construtos. Nesse sentido, é plausível supor, por exemplo, que uma pessoa que confia em sua capacidade para regular emoções, tanto em si quanto em outras pessoas, tenha, por isso mesmo, mais capacidade para ultrapassar obstáculos, tomar decisões e resolver problemas (percepção de si mesmo). Tal explicação é compatível com a afirmação de que pessoas resilientes tendem a fazer uso de emoções positivas para lidar com fatores estressantes (Tugade \& Fredrickson, 2007).

Os resultados deste estudo também apoiam a concepção de Troy e Mauss (2011) e de Masten e Obradovic (2006), que propuseram que a habilidade de regular emoções seria um moderador cognitivo, de tal forma que as pessoas com alta capacidade de RE cognitiva devem apresentar aumento da probabilidade de serem resilientes após exposição ao estresse. Assim, uma pessoa com elevada habilidade de RE tenderia a ser mais resiliente do que outra com baixa habilidade de RE, o que pode ser constatado nas análises correlacionais do presente estudo. Os resultados encontrados por Min et al. (2013) e New et al. (2009) com pacientes ansiosos/depressivos e vítimas de violência sexual, respectivamente, apoiam essa hipótese da RE como um importante mecanismo pelo qual uma pessoa se torna resiliente e consegue enfrentar de maneira mais efetiva o estresse decorrente de suas condições psicológicas.

Assim, esses resultados apoiam a ideia de que a resiliência e a RE podem ser consideradas construtos distintos, correlacionados positivamente entre si e que, possivelmente, apresentam um processo em comum: a percepção. Uma das implicações para a pesquisa é que 
esses construtos devem ser investigados separadamente, pois apresentam aspectos únicos que os distinguem entre si. A resiliência apresenta escopo mais amplo, envolvendo outros aspectos além do processamento emocional, como redes sociais e familiares, e também aspectos mais relacionados com a personalidade, como estilo estruturado, cuja definição se assemelha muito à do traço de conscienciosidade do modelo dos cinco grandes fatores.

De fato, as concepções atuais de resiliência tendem a compreendê-la como resultante de um conjunto de características cognitivas, emocionais, comportamentais e sociais que favorecem a adaptação diante de eventos estressores (Hjemdal et al., 2009). Já a RE parece se constituir em uma habilidade mais restrita ao processamento de informações emocionais (IE), que pode estar implicada tanto na resistência e na recuperação do estresse quanto em outras situações relacionais não necessariamente ligadas ao estresse.

Assim, este estudo contribui para a ciência psicológica ao apresentar evidências empíricas que apoiam a distinção entre os construtos. No entanto, é preciso apontar algumas limitações metodológicas que têm implicações para a generalização dos resultados encontrados neste trabalho. Uma delas se refere à distinção entre os instrumentos e os construtos. Os instrumentos empregados neste estudo se constituem em operacionalizações das variáveis estudadas, mas podem enfatizar um ou outro aspecto delas e omitir ou amenizar outros. Por isso, a generalização da discriminação entre as medidas encontradas nesta pesquisa para os respectivos construtos deve ser feita com cautela. Seria interessante, por exemplo, que estudos semelhantes fossem realizados com instrumentos diferentes para a avaliação das mesmas variáveis, de modo a checar se os achados se confirmam.

Outra limitação importante se refere à amostra, que foi constituída predominantemente por mulheres nordestinas com nível de escolaridade superior. Para que a generalização para a população seja feita com mais segurança, é necessário replicar o estudo com uma amostra distinta, mais estratificada quanto a aspectos sociodemográficos e de nível de escolaridade e equitativa quanto à distribuição dos sexos dos participantes. Apesar disso, o estudo contribui para o desenvolvimento da ciência psicológica, não apenas por apresentar evidências da distinção entre as variáveis, mas também por identificar um ponto em comum - a percepção, que também merece ser investigada em trabalhos futuros.

\section{Referências}

Bueno, J. M. H., Correia, F. M. de L., Abacar, M., Gomes, Y. de A., \& Pereira Jr., F. S. (2015). Competências emocionais: estudo de validação de um instrumento de medida. Avaliação Psicológica, 14(1), 153-163. doi: 10.15689/ap.2015.1401.17.

Carvalho, V. D., Teodoro, M. L. M., \& Borges, L. O. (2014). Escala de Resiliência para Adultos: aplicação entre servidores públicos. Avaliação Psicológica, 13(2), 287-295. Recuperado de http://www.redalyc.org/html/3350/335031819016/

Cicchetti, D. (2013). An overview of developmental psychopathology. In P. D. Zelazo (Ed.), The Oxford handbook of developmental psychology. (pp. 455-480). New York: Oxford University Press.

Correia, F. L. \& Bueno, J. M. H. (2014). Estudo de validação de Instrumento para Avaliação das Habilidades Emocionais. In VII Congresso Brasileiro de Avaliação Psicológica, 2015, São Paulo. Anais do VII Congresso Brasileiro de Avaliação Psicológica.

Cruvinel, M., \& Boruchovitch, E. (2011). Regulação emocional em crianças com e sem sintomas de depressão. Estudos de Psicologia, 16(3), 219-226. Recuperado de http://www.redalyc.org/html/261/26122323003/

Darwin, C. R. (1872). The expression of the emotion in man and animals. London: John Murray.

Evans, G. W., Li, D., \& Whipple, S. S. (2013). Cumulative risk and child development. Psychological Bulletin. 139(6), 1342-1396. doi: 10.1037/a0031808

Freud, A. (1946). The ego and the mechanisms of defense. New York: International Universities Press.

Gross, J. J. (2002). Emotion regulation: Affective, cognitive, and social consequences. Psychophysiology, 39(3), 281-291. doi: 10.1017. S0048577201393198

Gross, J. J (2008). Emotion regulation. Em M. Lewis, J. M. Haviland-Jones, \& L. F. Barrett (Eds.), Handbook of emotions. New York: Guilford Press, $3^{\mathrm{a}}$ ed., 497-512.

Gross.J.J. (2015). Emotion Regulation: Current Status and Future Prospects. Psychological Inquiry, 26(1), 1-26. doi: 10.1080/1047840X.2014.940781

Hair, J. F., Black, W. C., Babin, B. J., Anderson, R. E., \& Tatham, R. L. (2009). Análise multivariada de dados. Porto Alegre: Bookman.

Harris, A., Hare, T., \& Rangel, A. (2013). Temporally dissociable mechanisms of self-control: early attentional filtering versus late value modulation. Journal of Neuroscience, 33(4), 18917-18931. doi: 10.1523/JNEUROSCI.5816-12.2013

Hjemdal, O., Friborg, O., Martinussen, M., \& Rosenvinge, J. H. (2001). Preliminary results from the development and validation of a Norwegian scale for measuring adult resilience. Journal of the Norwegian Psychological Association, 38(4), 310-317. doi: 10.1186/ s40359-015-0076-1

Hjemdal, O., Roazzi, A., Dias, M. G. B. B., Roazzi, M., \& Vikan, A. (2009). Exploring the psychometric properties of the resilience scale for adults in a Brazilian sample. In Elizur, D., \& Yaniv, E. (Eds.), Facet new horizons in theory construction and data analysis (pp. 120-138). Jerusalem: FTA. doi: 10.17652/rpot/2015.1.349

Junqueira, M. F. P. S., \& Deslandes, S. F. (2003). Resiliência e maus-tratos à criança. Cadernos de Saúde Pública, 19(1), 227-235. doi: 10.1590/ S0102-311X2003000100025 
Karreman, A., \& Vingerhoets, A. J. (2012). Attachment and well-being: the mediating role of emotion regulation and resilience. Personality and Individual differences, 53(7), 821-826. doi: 10.1016/j.paid.2012.06.014

Kemper, T. D. (2004). Social models in the explanation of emotions. In Lewis, M., \& Haviland-Jones, J. M. (Eds.), Handbook of emotions (2a ed., pp. 45-58). New York: Guilford.

Knorst, C. E. K. (2012). Resiliência: instrumentos de avaliação no contexto brasileiro (Monografia de Especialização em Psicologia). Universidade Federal do Rio Grande do Sul, Porto Alegre.

Koller, S. H., Morais, N. A., \& Cerqueira-Santos, E. (2009). Adolescentes e jovens brasileiros: levantando fatores de risco e proteção. Em R. M. C., Libório \& S. H., Koller (Eds), Adolescência e Juventude: risco e proteção na realidade brasileira (pp. 17-56). São Paulo, SP: Casa do Psicólogo.

Lazarus, R. S. (1996). Psychological stress and the coping process. New York: McGraw Hill.

MacCann, C., Joseph, D. L., Newman, D. A., \& Roberts, R. D. (2014). Emotional intelligence is a second-stratum factor of intelligence: Evidence from hierarchical and bifactor models. Emotion, 14(2), 358-374. doi: 10.1037/a0034755

MacCann, C., \& Roberts, R. D. (2008). New Paradigms for Assessing Emotional Intelligence: Theory and Data. Emotion, 8(4), $540-551$. doi: $10.1037 / \mathrm{a} 0012746$.

Masten, A. S. (2013). Risk and resilience in development. In P. D. Zelazo (Ed.), Oxford handbook of developmental psychology (pp. 579-607). New York: Oxford University Press. doi: 10.1093/oxfordhb/97800199958474.013.0023.

Masten, A. (2014). Global Perspectives on Resilience in Children and Youth. Child Development, 85(1), 6-20. doi: 10.1111/cdev.12205

Masten, A. S., \& Obradović, J. (2006). Competence and resilience in development. Annals of the New York Academy of Sciences, $1094(1), 13-27$.

Mayer, J. D., Caruso, D. R., \& Salovey, P. (2016). The Ability Model of Emotional Intelligence: Principles and Updates. Emotion Review, 8(4), 290-300. doi: 10.1177/1754073916650500

Mayer, J. D., \& Salovey, P. (1997). What is Emotional Intelligence? In P. Salovey, \& D. J. Sluter (Eds.), Emotional Development and Emotional Intelligence. New York: Basic Books.

Mayer, J. D., Salovey, P., \& Caruso, D. R. (2002). Mayer-Salovey-Caruso Emotional Intelligence Test (MSCEIT) User's Manual. Toronto: Multi-Health Systems.

Min, J., Yu, J. J., Lee, C., \& Chae, J. (2013). Cognitive emotion regulation strategies contributing to resilience in patients with depression and/or anxiety disorders, Comprehensive Psychiatry, 54(8), 1190-1197. doi: 10.1016/j.comppsyck.2013.05.008

New, A. S., Fan, J., Murrough, J. W., Liu, X., Liebman, R. E., Guise, K. G. Tang, C.Y., \& Charney, D. S. (2009). A Functional Magnetic Resonance Imaging Study of Deliberate Emotion Regulation in Resilience and Posttraumatic Stress Disorder. Biological Psychiatry, 66(7), 656-664. doi: 10.1016/j.biopsych.2009.05.020

Overton, W. F. (2013). A new paradigm for developmental science: Relationism and relational-developmental systems. Applied Developmental Science, 17(2), 94-107. doi: 10.1080/10888691.2013.778717

Paludo, S., \& Koller, S. H. (2005). Resiliência na rua: um estudo de caso. Psicologia: Teoria e Pesquisa, 21(2), 187-195. Recuperado de http:// www.scielo.br/pdf/ptp/v21n2/a09v21n2

Paula Couto, M. C. P., Poletto, M., Paludo, S., \& Koller, S. H. (2006). Resiliência e psicologia positiva. Em Facultad de Ciências Sociales de la Universidad de Palermo (Org.), Memórias del primer encuentro iberoamericana de psicologia positiva (p.50). Buenos Aires: AR.

Primi, R. (2003). Inteligência: avanços nos modelos teóricos e nos instrumentos de medida. Avaliação Psicológica, 2(1), 67-77. Recuperado de http://pepsic.bvsalud.org/pdf/avp/v2n1/v2n1a08.pdf

Reppold, C. T., Mayer, J. C., Almeida, L. S., \& Hutz, C. S. (2012). Avaliação da Resiliência: Controvérsia em Torno do Uso das Escalas. Psicologia: Reflexão e Crítica, 25(2), 248-255. Recuperado de http://www.scielo.br/pdf/prc/v25n2/a06v25n2

Salovey, P., \& Mayer, J. D. (1990). Emotional Intelligence. Imagination, Cognition and Personality, 9(3), 185-211. doi: 10.2190/DUGG-P24E$52 \mathrm{WK}-6 \mathrm{CDG}$

Schneider, T. R., Lyons, J. B., \& Khazon, S. (2013). Emotional intelligence and resilience. Personality and Individual Differences, 55(8), 909-914. doi: 10.1016/j.paid.2013.07.460

Troy, A. S. \& Mauss, I. B. (2011). Resilience and Mental Health: Challenges Across the Lifespan. In S. M. Southwick, B. T. Litz, D. Charney, \& M. J. Friedman. Cambridge: Cambridge University Press.

Tugade, M. M., \& Fredrickson, B. L. (2007). Regulation of positive emotions: Emotion regulation strategies that promote resilience. Journal of Happiness Studies, 8(3), 311-333. doi: 10.1007/s10902-006-9015-4

Wundt, W. (1863). Lectures on the minds of men and animals. Leipzig: Engelmann.

\section{Sobre os autores}

Angélica Maria Ferreira de Melo Castro é psicóloga. Mestre em Psicologia do Desenvolvimento Humano pela Universidade Federal de Minas Gerais (UFMG). Doutoranda no Programa de Pós Graduação em Psicologia Cognitiva da Universidade Federal de Pernambuco (UFPE), sendo bolsista CNPq e pesquisadora do Núcleo de Estudos em Avaliação Psicológica da UFPE (NEAP/UFPE).

José Maurício Haas Bueno é psicólogo. Doutor em Psicologia pela Universidade São Francisco (USF). Professor do Programa de Pós Graduação em Psicologia Cognitiva da UFPE e coordenador do NEAP/UFPE.

Mirela Dantas Ricarte é psicóloga. Mestre e doutoranda em Psicologia Cognitiva na UFPE. Pesquisadora do NEAP/UFPE.

Emily Souza Gaião e Albuquerque é psicóloga. Mestre e doutoranda em Psicologia Cognitiva pela UFPE. Bolsista CNPq. Pesquisadora do NEAP/UFPE. Professora substituta do departamento de Psicologia da Universidade Estadual da Paraíba (UEPB). 